\title{
Enfoque metodológico para los objetivos estratégicos en la planificación del sector público
}

\section{RESUMEN}

El presente artículo se sustenta en el análisis de los objetivos estratégicos establecidos en los planes de las diferentes instituciones locales, regionales y nacionales del sector público, con la finalidad de determinar la cantidad de objetivos y calidad en la redacción de los mismos; además de proponer mejoras metodológicas para tal fin.

Palabras clave: objetivos estratégicos, planes estratégicos, planificación, sector público

METHODOLOGiCal APPROACH FOR STRATEgic OBJECTIVES IN PLANNING PUBLIC SECTOR

\section{ABSTRACT}

This article is based on the analysis of the strategic objectives set in the plans of the various local, regional and national public sector institutions, in order to determine the amount and quality objectives in drafting them; also proposing methodological improvements for this purpose.

Keywords: strategic objectives, strategic plans, planning, public sector

\section{INTRODUCCIÓN}

La importancia del Plan Estratégico Institucional es vital para los administradores de una organización, ya que en ella se plasma la dirección que le quieren dar a la institución, y cuando es transmitido en forma adecuada, genera sinergias en todo el personal para la obtención de sus objetivos.

El sector público en nuestro medio es uno de los que utiliza como sustento de gestión a los planes estratégicos en los tres niveles de gobierno: nacional, regional y local. La esencia de todo plan estratégico son los cambios que se desean lograr en el futuro expresado en objetivos; cuya propuesta determinan el accionar de toda institución púbica; de allí la importancia del tema.

La finalidad del estudio es analizar en los planes institucionales de los tres niveles de gobierno, la coherencia que existe en los objetivos estratégicos en cuanto a la cantidad y su calidad.

\section{SISTEMA NACIONAL DE PLANEAMIENTO ESTRATÉGICO (SINAPLAN)}

El Sistema Nacional de Planeamiento Estratégico es el conjunto articulado e integrado de órganos, subsistemas y relaciones funcionales cuya finalidad es coordinar y viabilizar el proceso de planeamiento estratégico nacional para promover y orientar el desarrollo armónico y sostenido del país.

\section{Ente rector}

El Centro Nacional de Planeamiento Estratégico -CEPLAN-, como organismo de derecho público se constituye como el órgano rector y orientador del Sistema Nacional de Planeamiento Estratégico. Sus competencias son de alcance nacional y constituye un pliego presupuestario.

El Centro Nacional de Planeamiento Estratégico asume las funciones relacionadas al planeamiento estratégico y evaluación, que cumple la Dirección General de Programación Multianual del Sector Público del Ministerio de Economía y Finanzas y las de seguimiento y evaluación estratégica del Estado que cumple la Secretaría de Gestión Multisectorial de la Presidencia del Consejo de Ministros.

* Magíster en Gestión Económica y Empresarial, Ingeniero Industrial de la UNMSM. Profeso en la Facultad de Ingeniería Industrial, Departamento Académico de Producción y Gestión Industrial de la UNMSM. E-mail: cieandia@hotmail.com 
La directiva $\mathrm{N}^{\circ}$. 001-2014-CEPLAN, referida al proceso de planeamiento estratégico, establece la siguiente tipología de planes:

- El Plan Estratégico Sectorial Multianual (PESEM)

- Plan de Desarrollo Regional Concertado (PDRC)

- Plan de Desarrollo Local Concertado (PDLC)

- Plan Estratégico Institucional (PEI)

- Plan Operativo Institucional (POI)

- Planes Especiales

\section{Planeamiento estratégico}

"Es el proceso por el cual los miembros guía de una organización prevén su futuro y desarrollan los procedimientos y operaciones necesarias para alcanzarlo" (Goodstein, 1998, p. 5).

Es un esfuerzo organizacional, definido y disciplinado que busca que las instituciones definan con la mayor claridad posible sus acciones para el logro de sus objetivos en el futuro.

El proceso de prever el futuro es muy diferente de la planeación a largo plazo; este, a menudo, es simplemente la extrapolación de tendencias actuales. Prever es más que tratar de anticipar el futuro y prepararse en forma apropiada; implica la convicción de que lo que hace la institución ahora puede influir en los aspectos del futuro y modificarlos.

La planeación estratégica debe responder tres preguntas básicas: a. ¿Cuál es su entorno?

b. ¿Hacia dónde va la institución?

c. ¿Cómo lograrlo?

Por lo tanto, todo plan busca establecer lo siguiente:

- Visión: ¿Cuál será el estado futuro de la institución?

- Objetivos: ¿Qué se desea lograr?

- Estrategias: ¿Cómo lograr los objetivos y qué alternativas se debe emplear?

- Metas: ¿Cómo saber que se ha logrado lo esperado?

- Políticas internas: ¿Qué orientación y prioridades deben alcanzarse?

- Procedimientos: ¿Cómo se hará?

- Normas de control: ¿Qué parámetros y/o medidas se utilizan en la evaluación?

Por tanto, todo plan estratégico produce beneficios relacionados con la capacidad de realizar una gestión más eficiente bajo un lenguaje común, utilizando eficientemente los recursos humanos y materiales para el logro de los objetivos.

\section{Ejes estratégicos}

Son aquellos enfoques que agrupan los servicios y/o funciones de la institución y delinean las acciones y sustentan la razón de ser de una institución a través de sus objetivos, estrategias, indicadores, entre otros aspectos; además, integran conceptualmente la misión y visión (Andía, 2011, p. 89).

Figura 1. Los ejes estratégicos en la planificación.

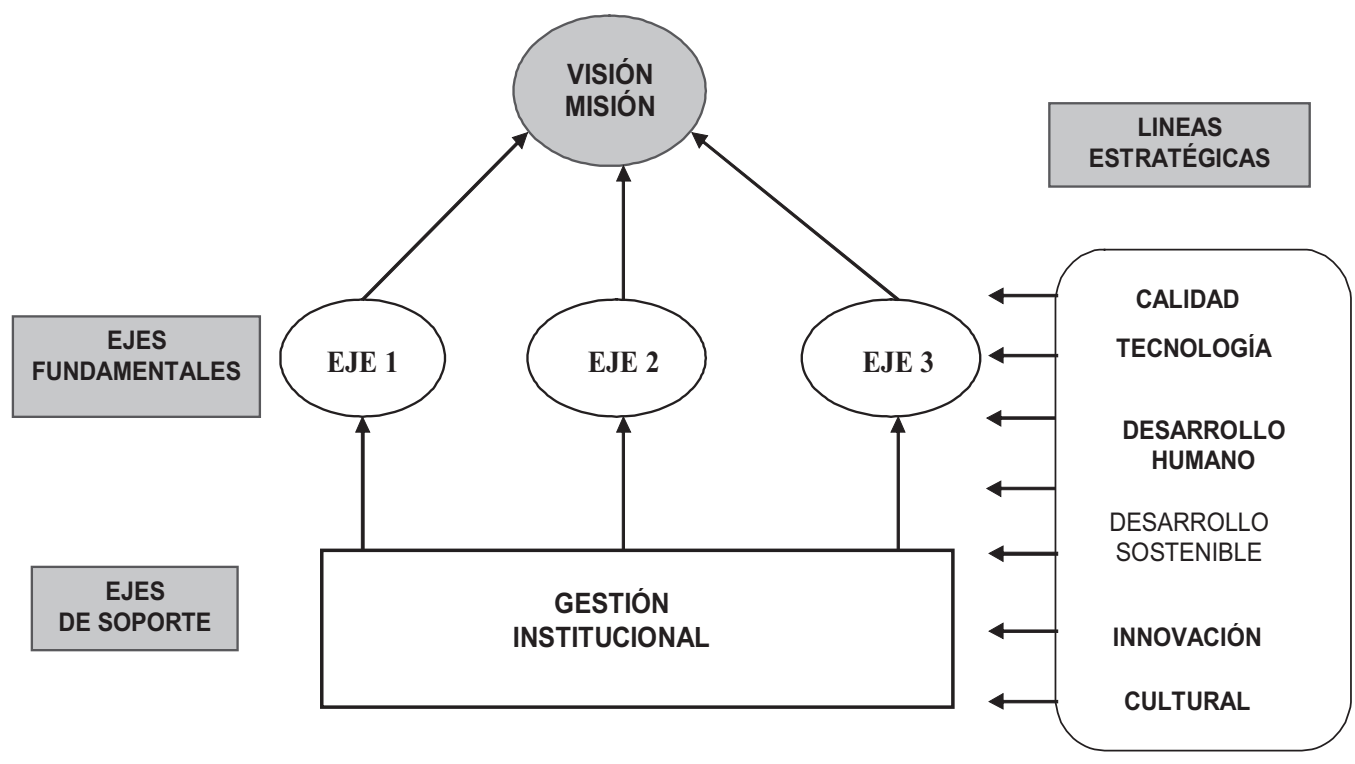


Los ejes estratégicos se dividen en ejes fundamentales y ejes de soporte; la Figura 1 muestra su integración con la planificación. En una institución, se recomienda que la cantidad máxima de ejes estratégicos sea cuatro, uno de soporte y tres como servicios fundamentales que brinda la institución e integra todo sus actividades.

\section{Objetivos estratégicos}

Los objetivos estratégicos son, por definición, objetivos de mediano y largo plazo, orientados al logro de la misión de la organización. Son los resultados más relevantes y de mayor nivel que la institución espera lograr para cumplir con su misión.

Lira (2006) sostiene que la planificación estratégica debe proponer el logro de objetivos que sean coherentes en el largo, mediano y corto plazo y trata de cumplirlos con los menores costos posibles.

Por tanto, los objetivos estratégicos deben ser siempre cuantificables, realizables en cantidad y calidad, comprensibles, estimulantes, coherentes y escritos en forma jerárquica preferentemente.

- Cuantificables: permiten expresar en valores cuantitativos lo que se desea lograr, ejemplo: el objetivo para una empresa es incrementar su participación de mercado de un $10 \%$ a $15 \%$ en tres años. El objetivo establece el cambio y el momento.

- Realizables: los objetivos deben ser posibles de alcanzar. No tiene sentido diseñar un objetivo estratégico que de antemano se sabe que no se podrá lograr.

- Comprensibles: se refiere a que deben estar escritos con palabras muy sencillas y deben de ser comprendidos fundamentalmente por quienes están involucrados en su logro.

- Motivadores: deben estimular a la mayor productividad.

\section{Metodología del estudio}

Para obtener información sobre el tema del estudio, se revisaron un conjunto de planes estratégicos de los tres niveles de gobierno: nacional, regional y local. En total se analizaron 51 planes estratégicos, distribuidos en: 14 planes del gobierno nacional (específicamente ministerios); 10 planes de los gobiernos regionales y 27 planes de los gobiernos locales. Para cada uno de los planes se obtuvo la cantidad de objetivos estratégicos propuestos en dichos documentos. El resumen de la información encontrada se presenta en el siguiente cuadro:
Cuadro 1. Objetivos Estratégicos.

\begin{tabular}{|c|c|c|c|}
\hline \multicolumn{4}{|c|}{ Resumen } \\
\hline $\begin{array}{c}\text { N. }{ }^{\circ} \text { de } \\
\text { objetivos } \\
\text { estratégicos }\end{array}$ & $\begin{array}{c}\text { Gobierno } \\
\text { nacional }\end{array}$ & $\begin{array}{c}\text { Gobierno } \\
\text { regional }\end{array}$ & $\begin{array}{c}\text { Gobierno } \\
\text { local }\end{array}$ \\
\hline$[0-4]$ & 8 & 0 & 5 \\
\hline$<4-10]$ & 5 & 9 & 13 \\
\hline$<10-$-a más] & 1 & 1 & 9 \\
\hline Total & 14 & 10 & 27 \\
\hline
\end{tabular}

Fuente: Elaboración propia.

Dicha información se puede interpretar de la siguiente manera:

- De un total de 14 instituciones del gobierno nacional hay 6 instituciones (43\%) que tienen más de 4 objetivos estratégicos (específicamente más de 6 objetivos).

- En cuanto a los gobiernos regionales, las 10 instituciones (100\%) tienen más de 4 objetivos estratégicos. Son 7 instituciones con más de 7 objetivos.

- En los gobiernos locales de un total de 27 instituciones, 22 instituciones (81\%) tienen más de 4 objetivos estratégicos; de ellos, existen 15 instituciones con más de 7 objetivos en cada plan. Es en este sector donde se proponen la mayor cantidad de objetivos estratégicos.

Como menciona Sánchez (2003): la integración de cada uno de los planes operativos con la misión y los objetivos estratégicos, constituye el Plan General de Operaciones de la organización. Entonces, la cantidad de objetivos estratégicos es uno de los aspectos más relevantes en la planificación, si son muy pocos, se corre el riesgo de no incorporar los cambios en servicios significativos de la institución; si son muchos, se diluyen los logros que se desean alcanzar.

Frente a ello, se recomienda un objetivo por cada eje estratégico, es decir, considerando cuatro ejes en la gestión de una institución, se tendría cuatro objetivos estratégicos.

Por tanto, del análisis de la información, se tiene que en los tres niveles de gobierno gestionan sus planes con una cantidad excesiva de objetivos estratégicos, lo cual repercute en los resultados y logros de sus metas. 


\section{Análisis Cualitativo}

Desde el punto de vista cualitativo, se encontró que la redacción de los objetivos es inadecuada en los planes estratégicos de los tres niveles de gobierno, se puede mencionar los siguientes casos:

- Se proponen objetivos que son funciones de la institución, por tanto, no hay logros que medir en el futuro.

- Objetivos que no están orientados hacia resultados, sino más a acciones y tareas.

- Se observa dificultades de las diferencias entre objetivos y metas.

- Se proponen muchos objetivos que no son de transformación y crecimiento de la organización, sino más bien de mantenimiento del sistema.

- Objetivos poco concretos y ambiguos.

- No se presentan alineamiento y coherencia entre los objetivos.

Alguno de los casos de objetivos inadecuadamente planteados se muestra como ejemplo a continuación:

- Facilitar la igualdad de oportunidades para la población impulsando la descentralización e integración macro regional, y el respeto de la diversidad cultural de la población (ambiguo).

- Atender de manera oportuna, predecible y confiable los servicios, las solicitudes y las denuncias presentadas ante la institución por nuestros usuarios (función).

- Garantizar la seguridad y el orden interno con eficiencia y capacidad preventiva, buscando el acercamiento a la comunidad y respetando los derechos civiles y políticos (función).

- Priorizar las intervenciones de prevención de las enfermedades transmisibles y no transmisibles promoviendo estilos de vida y entornos saludables (Tarea).

- Garantizar la seguridad y el orden interno con eficiencia y capacidad preventiva, buscando el acercamiento a la comunidad y respetando los derechos civiles y políticos (mantenimiento del sistema y función).

- Proteger, en las actividades de competencia del Sector MYPE e Industria, el ambiente, la salud y la seguridad de las personas, así como contribuir en la lucha contra los delitos aduaneros y delitos contra la propiedad intelectual (ambiguo).

\section{Propuesta metodológica para establecer objetivos}

1. Definir los ejes estratégicos

Se recomienda que la cantidad máxima de ejes estratégicos sea cuatro, uno de soporte y tres como servicios fundamentales. Ello evita la duplicidad e incluir funciones como objetivos, además, permite el alineamiento con su misión.

\section{Cantidad de objetivos}

Por sus características los objetivos estratégicos deben ser uno por cada eje establecido; es decir, un total de cuatro.

3. Redacción de los objetivos estratégicos

Para mejorar la redacción de los objetivos se tienen dos enfoques. El primero es expresarlos como productos o resultados esperados; el segundo se denomina "objetivos más".

\subsection{Los objetivos como productos o resultados}

Lo que se debe destacarse es lo que se desea lograr; es decir, el objeto resultante de los esfuerzos que se deben realizar. La práctica más generalizada en nuestro medio para redactar los objetivos es iniciar la frase con un verbo.

Aunque esta forma de redactar los objetivos resulta más natural, tiene el inconveniente de que en los procesos de análisis y planificación estratégica puede llevar a confusiones entre objetivos y actividades. Para evitar tales confusiones es recomendable reservar el uso de los verbos infinitivos para enunciar las actividades con las cuales se propone alcanzar los objetivos.

\section{Ejemplo:}

"Mejorar la oferta de los servicios de salud en beneficio de la población en general."-Plan Estratégico del Gobierno de Junín

\subsection{Los objetivos más}

Se les llama así porque se construyen con expresiones tales como "más" o "menos" o algunos sinónimos tales como "mejora", "incremento", "reducción", "disminución". Algunas frases típicas pueden ser: "Mejor servicio..., más eficiente..., más rápido..., mayor cantidad..., menos trámites...". Este tipo de objetivos debe expresar el objeto de cambio, un aspecto sobre la calidad y la dirección del cambio y el contexto, lugar o ambiente en el cual se realiza el objetivo propuesto.

Objetivo para el ejemplo anterior corregido: 
"Mejor oferta de los servicios de salud en beneficio de la población en general."

\section{CONCLUSIONES}

1. En cuanto a la calidad, los objetivos no reúnen los criterios que los identifican como tal, por ello, existe duplicidad de metas, no pueden ser evaluados, se confunden con las funciones y actividades de la institución.

2. En cuanto al análisis cuantitativo de los objetivos estratégicos encontrados en los planes, no se puede determinar una relación sobre la cantidad de los mismos en los tres niveles de gobierno.

3. Como propuesta metodológica, la cantidad de objetivos estratégicos en la planificación deben guardar relación con los ejes estratégicos definidos en la institución.

\section{REFERENCIAS BIBLIOGRÁFICAS}

[1] Andía, W., (2011). Planeamiento estratégico en el sector público. 2da. edición. El Saber Editores. Perú.

[2] Goodstein, L. (1998). Planeación estratégica aplicada, Mc Graw Hill. Colombia,

[3] Lira, L. (2006). Revalorización de la planificación del desarrollo. Instituto Latinoamericano y del Caribe de Planificación Económica Social. Chile.

[4] Sánchez, F. (2003). Planificación estratégica y gestión pública por objetivos. Instituto Latinoamericano y del Caribe de Planificación Económica Social. Chile. 